\title{
Origins of life
}

\author{
AUGUSTO DAMINELI \\ ↔ DANIEL SANTA CRUZ DAMINELI
}

\begin{abstract}
What is life?
A lthough the word life seems to have an obvious meaning, it leads to different ideas, making it necessary for us to define the very object to which we refer in this text. For psychologists, it brings to mind the psychic life; for sociologists, the social life; for theologians, the spiritual life; for the common people, the pleasures or the sore spots of existence. This is part of our strongly anthropocentric view of the world. For a (relatively small) part of the people, it brings to mind images of forests, birds and other animals. Even that image is partial, since the great majority of the living organisms are invisible. The microbes constitute the greatest part of living organisms, most of which $(80 \%)$ living below the Earth's surface, adding up to a mass equal to that of plants. However, microbes still do not occupy the due dimension in our imaginary, despite more than a century of use of the microscope and of frequent news in the media involving the powerful action of microbes, sometimes causing diseases and other times curing them, being a part of the ecosystem or affecting food production. That framework is due to the fact that life is still a recent theme in the scientific realm, compared to its ancientness in the philosophic and religious thought.
\end{abstract}

A broadly divulged conception among people of a Jewish-ChristianIslamic culture is that God breathed life into matter and that, therefore, it was a kind of miracle instead of deriving from natural laws. It is hard to trace the origin of that conception, but Aristotle's writings (384-322 B.C.) mention the pneuma, a kind of divine matter that supposedly constituted animal life. Pneuma was an intermediate stage of perfection right below that of the human soul. The duality between matter and life in the animals (or between body and soul in the human beings) was already present in the Socratic school, of which Aristotle was a member, although in a somewhat different manner. Among the superior animals, the breath of life was passed on to the descendents through reproduction. However, Aristotle believed that some creatures (insects, eels, oysters) appeared spontaneously, without deriving from the "seed" of another living creature. That concept is known as spontaneous generation and seems to have derived from the pre-Socratic thinkers, who imagined that life, as well as all diversity in 
the world, was made up of a few basic elements. The idea of spontaneous generation is also present in ancient writings in China, India, Babylon and Egypt, as well as in other writings throughout the twenty centuries that followed, such as in authors like van Helmont, W. Harvey, Bacon, Descartes, Buffon and Lamarck. It seems Aristotle can be considered responsible for its dispersion throughout the Western world, due to the great influence he had on our culture.

A laboratory experiment by Louis Pasteur (1822-1895) put an end to the idea of spontaneous generation. After him, people began to admit that life could only come from another life. Curiously enough, however, Pasteur used to say that he had not eliminated completely the possibility of spontaneous generation. Indeed, his experiment could not be applied to the first life form, and the idea that life could come from inorganic matter continued in the agenda among other great scientists. However, it changed to such a different context from the previous views that it cannot be labeled in the same way. That new kind of "spontaneous generation" would only be valid for the first life form. After that, reproduction would be required.

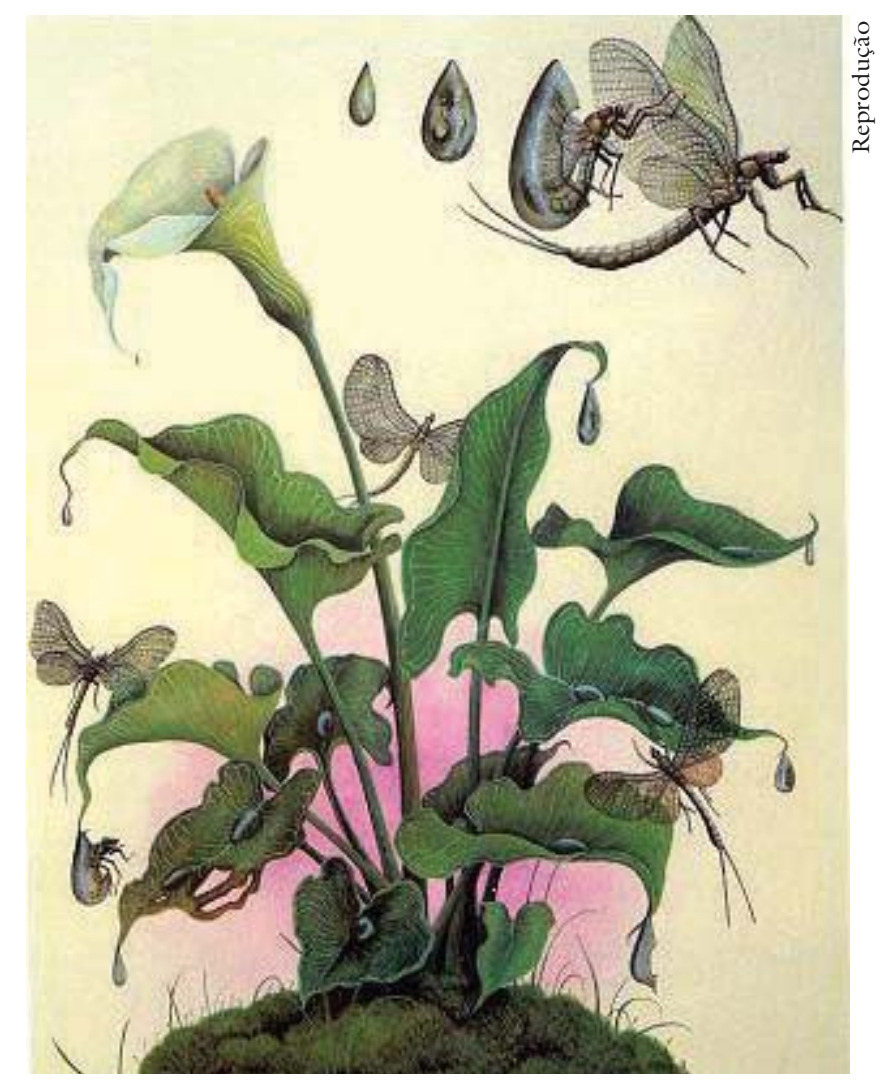

Figure 1 - Spontaneous generation for organisms considered to be simple was admitted since Ancient Times until the end of the 19th Century. 
Charles Darwin (1809-1882) believed that a puddle of primeval soup, containing ammonia, phosphorus salts, light, heat and electricity could have created proteins that were transformed in more complex compounds, until they originated living organisms. However, the extension of the evolution to the molecular world as the first chapter of the evolution of life only developed beginning with the ideas of Alexander Ivanovich Oparin (1894-1980). He tried to understand the origin of life as part of the biochemical reactions' evolution, by means of the Darwinian competition and selection, in the pre-biotic Earth (before the emergence of life).

As for the place where life was supposed to exist, the Vedas and Upanishads in India believed in the existence of life particles that permeated the entire Universe. Anaxagoras ( 500-428 B.C.) also believed that life was present in the entire cosmos. During the Renaissance, Giordano Bruno enthusiastically preached the existence of other inhabited worlds. The analysis of meteorites made by Berzelius during the 1830's showed the existence of organic compounds in space. Based on that, the physicist and chemist Savante A. Arrhenius (1859-1927) suggested that, besides organic products, life itself had originated in space, being transported here by meteorites. Versions of that idea were presented by Richter, Kelvin, Chamberlain and, more recently, by Francis Crick, Fred Hoyle, Chandra Wickramasinghe, John Oró and others. Not even the advocates of that hypothesis, called panspermia, or those of the competing scenario (according to whom life originated on Earth) presented solid proofs about where life began. Indeed, that is a secondary problem, compared to other more relevant issues.

Throughout the last century, the origin of life began to be approached in a scientific manner, by means of laboratory experiments and the study of theoretical processes. It became an eminently interdisciplinary theme, involving cosmology, astrophysics, planetology, geology, organic chemistry, molecular biology, mathematics and complex systems theory. In the last fifty years it was subdivided into several subthemes, some of which had remarkable progresses. Nevertheless, some key issues continue without a solution.

We do not even have a universally accepted concept of what life is. Why is a definition as something that is born, grows, reproduces itself and dies not sufficient to characterize life? Simply because several natural phenomena would fit into that definition. Think about something like a fire or a storm, or even some computer softwares. Such a definition does not help biologists at all and for that reason they do not depend on it. Instead of talking about "life" in a general manner, using the concept of living organism is much more operational. A living organism is based on the cell, in which the genetic information is codified in the DNA (deoxyribonucleic acid) and expresses itself in the form of proteins. It can be noticed that this concept is modern, having been developed after the invention of the microscope and the discovery of the 
genetic code. To reach that point, many modifications took place throughout history, as will be seen later.

It is in that specific context that life will be approached in this essay. But in a panorama with so many possibilities, why will we limit ourselves to discuss only the common kind of life that we know? Are there other kinds, here or in other planets? How can we recognize them? The restriction is not because we deny the possibility of the existence of other life paradigms, but because that is the only one that makes a scientific approach possible, because it presents observable data and theoretical models. Countless attempts to formulate a general concept of life were and are still being made, but none of them presented significant advantages to understand the life we know, nor did it predict the existence of still unknown forms that can be observed. A General Theory of Life has not been developed yet and that limits our capacity to understand it. Only the discovery of other life specimens independent from the one we know on Earth could lead us to broaden the conceptual horizons. In the final item, we will show projects that aim at discovering life outside the Earth and we will discuss its feasibility and its potential contribution to the understanding of life in the scientific realm.

\section{Evolution and life}

Evolution is the process of change in organisms throughout time, in such a way that those that exist today are different from the initial ones. Although there is a continuity chain throughout time, it is hard to infer the properties of the first organisms based on the current ones. Fossils enable us to recover some information about the body structure of the current species' ancestors. That allowed us to make an exuberant map of evolution throughout the last $\sim 540$ million years (M.a.). All the genetic phyla (body architectures) existent today appeared in the so-called "Cambrian explosion" that occurred around that time. It is characterized by the appearance of the multicellular organisms.

In the Pre-Cambrian (geological era older than 570 M.a.) the organisms were unicellular (made up of a single cell), which made both the formation of fossils and their discovery through microscopes much more difficult. Fossils of microorganisms were traced back to a past as distant as 3.5 billion years ago (B.a.). They are found in rocky aggregates that are still inhabited by bacterial colonies, the so-called stromatolites, ${ }^{1}$ such as those from Apex, a formation in Western Australia. They present eleven different kinds of fossils, showing how the cells divided and multiplied themselves (although there is some disagreement whether they are real fossils). Their shapes are not distinguishable from the current photosynthetic algae (cyanobacteria) that infest several environments on Earth. Even being primitive for current life, those fossils are from organisms so complex that they cannot have been the first life forms. Microbiologists and molecular 
biologists claim that cyanobacteria were one of the last great bacteria groups to appear.

How can we take our studies even further back in time? It is very hard to find rocks that are older than 3.5 billion years ago, since the surface of our planet is constantly recycled. The rocks from the surface are forced to plunge by the plate tectonics, and they are cooked under pressure deep beneath the surface. The older a rock, the rarer it is. Therefore, there is no hope of finding fossils much older than 3.5 billion years ago, which interrupts the path towards seeking the origin of life through that kind of registration.

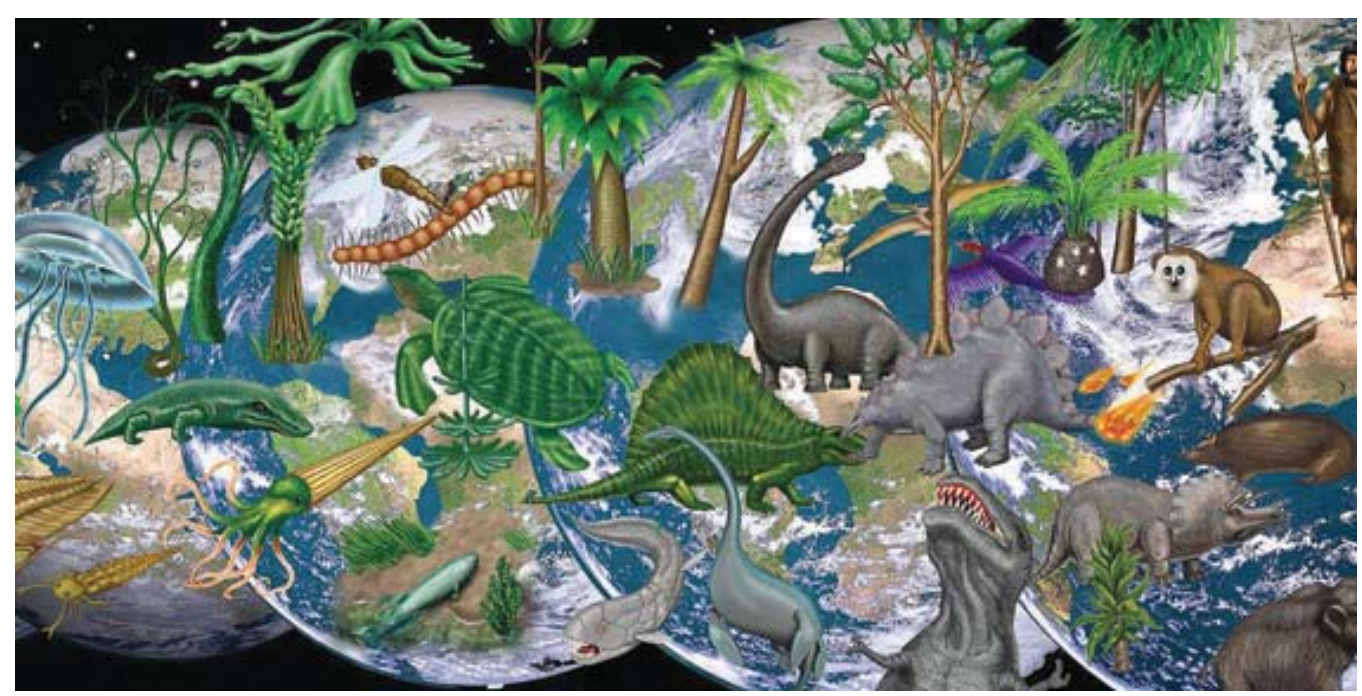

Figure 2 - The fossil record shows that evolution transformed the characteristics of living organisms throughout time, generating biological diversity and permitting the occupation of many niches available in the biosphere. (Art: Paulo Santiago)

Biological evolution is a surprising and unexpected fact when we have in mind that the genetic code works to make an exact copy of itself. The double helix structure is an extra guarantee of fidelity, providing two copies of each genetic information. If there were only forces that maintain the identity, biological diversity would not exist. However, there are processes that lead to imperfections in reproduction. Those are random "mistakes", natural in any copying process due to environmental radioactivity, cosmic rays coming from space or chemical agents. They generate copy-molecules that are different from the original ones in such a way that, when the molecule participates in the reproduction, the resultant organism will present (in general) small differences from its ancestor. If it adapts to the environmental conditions, it will survive and might leave descendants, increasing the biological diversity. 
There is no trend to produce more complex or more "perfect" organisms, as many people believe. The most complex ones do not seem to be more advantageous from the survival point of view as compared to simpler ones. If that was true, there would be far more complex organisms than simple ones, unlike what can be observed in nature. That idea of evolution as improvement is preached by the creationists, according to whom nature follows an intelligent design. They apply it not only to the biological evolution, but also to all natural phenomena. It is simply translated in the belief that the natural forces would not be able to create "order" and "beauty" if they were not guided by an intelligence that is external to matter itself. Adopting that point of view means giving science the role of merely finding out what that plan subjacent to nature is, which has already been predetermined forever. Such determinism was abandoned by Quantum Mechanics almost a century ago.
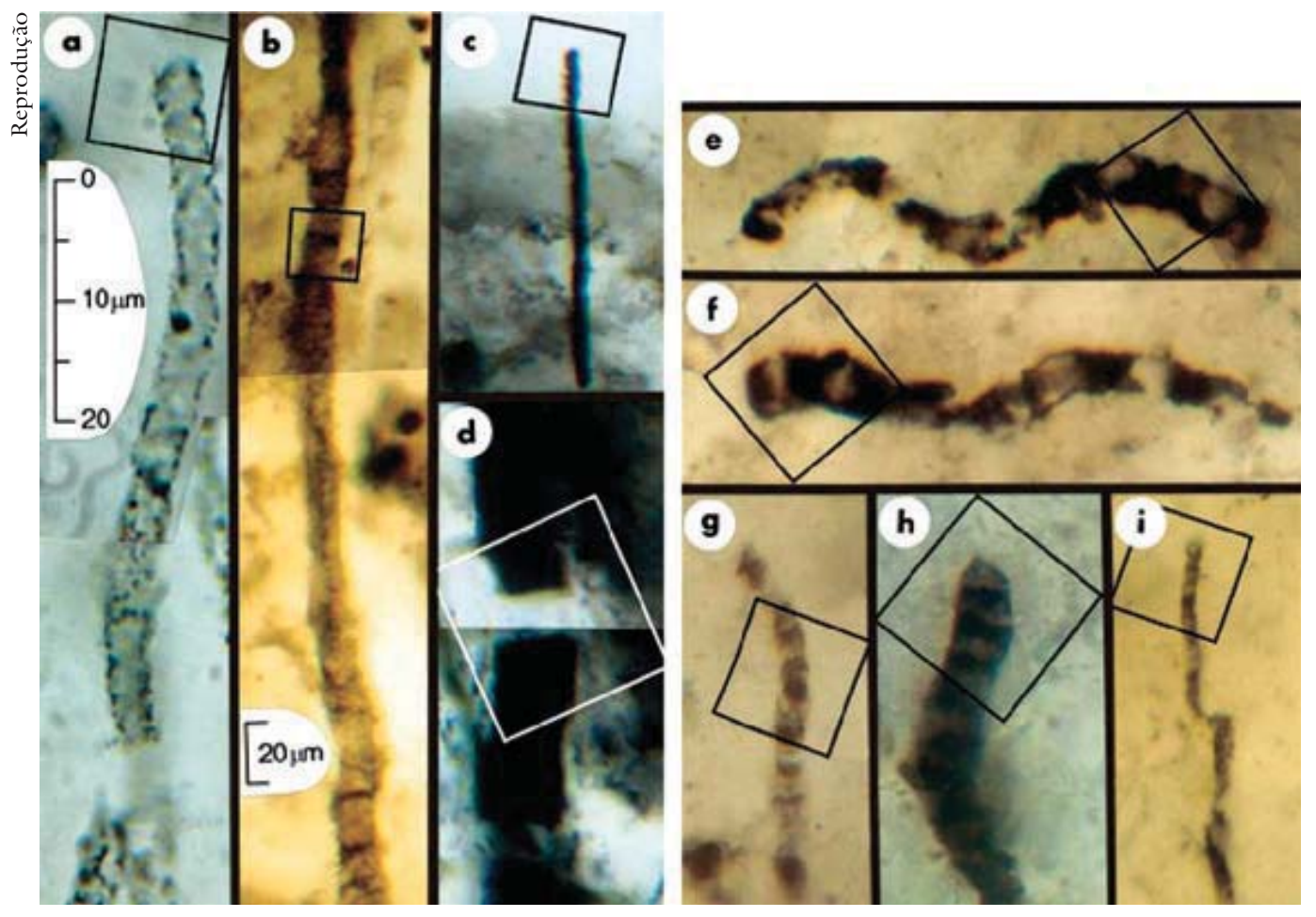

Figure 3 - Pre-Cambrian Microfossils. Specimens "e, f, g, h, i" are the oldest $(3,465$ B.a. $)$, found in the stromatolites of Apex, in Australia (J. W. Schopf et al. Nature, 416, 73, 2002).

\section{The universal tree of life}

Behind its huge variety of shapes, colors and sizes, the current organisms have very similar characteristics that can be used as important parameters to understand their origin. For example, water is the most abundant substance (molecule) of living matter: 70\% of the human body, 
$95 \%$ of the lettuce, $75 \%$ of bacteria. All organisms have a high percentage of water, which favors the hypothesis that their origin occurred in an aqueous environment. Its atomic composition is also admirably simple. Only four chemical elements - carbon, hydrogen, oxygen and nitrogen (CHON) - make up $99.9 \%$ of living matter. They are among the five most abundant in the Universe, leaving out only helium, which does not form chemical bonds. The biochemistry of life is made up of combinations of those atoms, forming water $\left(\mathrm{H}_{2} \mathrm{O}\right)$, methane $\left(\mathrm{CH}_{4}\right)$, ammonia $\left(\mathrm{NH}_{3}\right)$, carbon dioxide $\left(\mathrm{CO}_{2}\right)$, sugars, proteins, fatty acids and others. Although many proteins have metallic elements and require certain ions to operate, the most abundant elements are, by far, the ones mentioned above. The fact that life is made up of the most widely found atoms in nature indicates that it is simply an expression of the opportunity and not an exceptionality, a miracle, that could be done with arbitrary materials, including rare ones.

Table 1 - Abundance of chemical elements (in mass \%)

\begin{tabular}{l|c|c|c|c}
\hline Element & Bacteria & Mammals & Comets & Sun/Stars \\
\hline Hydrogen & 63 & 61 & 56 & 73.4 \\
\hline Oxygen & 29 & 26 & 31 & 0.8 \\
\hline Carbon & 6.4 & 10.5 & 10 & 0.2 \\
\hline Nitrogen & 1.4 & 2.4 & 2.7 & 0.09 \\
\hline Sulfur & 0.06 & 0.13 & 0.3 & 0.05 \\
\hline Phosphorus & 0.12 & 0.13 & 0.08 & 0.0007 \\
\hline
\end{tabular}

Although bacteria, whales, palm trees and elephants are so different from one another in shape, chemically speaking they are extremely similar. The simple molecules combine forming greater ones - the monomers, ${ }^{2}$ such as the nucleotides and the aminoacids. The nucleotides and aminoacids used by the living organisms are few and almost the same. The joining of those monomers in large chains constitutes the biopolymers: the nucleic acids (RNA and DNA) and proteins. They are responsible for the observable biological diversity. In the current living organisms, DNA carries the code to assemble proteins that are responsible for several functions. Besides the material composition, the form of energy processing (metabolism) is also very similar in all living organisms, occurring by means of a few intimately related processes. Figure 4 indicates that ALL living organisms are relatives and have a common origin.

A powerful way to diagnose the relationship among the living organisms is through genomic analysis ${ }^{3}$ of the $16 \mathrm{~S}$ ribosomal RNA, which cannot be applied to fossils, since they have lost their cellular content. The ribosomes are molecular complexes within the cells that participate in the production of proteins. Those protein factories are composed of many kinds of ribonucleic acid (RNA). Mutations, throughout time, change the order of the 
bases in the Ribosomal RNA (RNAr). Organisms belonging to a biological group that share a recent history have similar RNAr, and the more distant their relationship is, the more they differ. The comparison of $16 \mathrm{~S} \mathrm{RNAr}$ between two groups originated from a common evolutional branch allows us to assess how many changes took place since separation. Therefore, we can build a tree in which the length of the branch is proportional to the number of changes occurred, called the universal phylogenetic tree.

One can notice that, although plants and animals are the most familiar forms of life for us, they make up only two of the twenty branches of the tree of life. Besides those two, only fungi have members observable without a microscope. Most of life is invisible to the naked eye. All known organisms belong to one of the three domains: Bacteria (or Eubacteria), Archaea (or Archaebacteria) and Eukarya (or Eukaryotes). All branches connect to a single one in a region somewhere between Bacteria and Archea. That branch was supposedly the one of the last common ancestor (called progenote).

The phylogenetic tree is in conformity with the study of the fossils, which demonstrates that the oldest ones belonged to Bacteria and Archea domains, and the most recent ones belonged to Eucarya domain. It could be read as a time sequence, in which the present time would be in the tip of the branches and the past towards the connection with another branch. However, the division times of the branches cannot be measured accurately, since the mutation rates are not constant in time. Another aspect of the universal tree is that it is based on the organisms that are alive today, which account for less than $1 \%$ of all species that occurred in the long history of the planet.

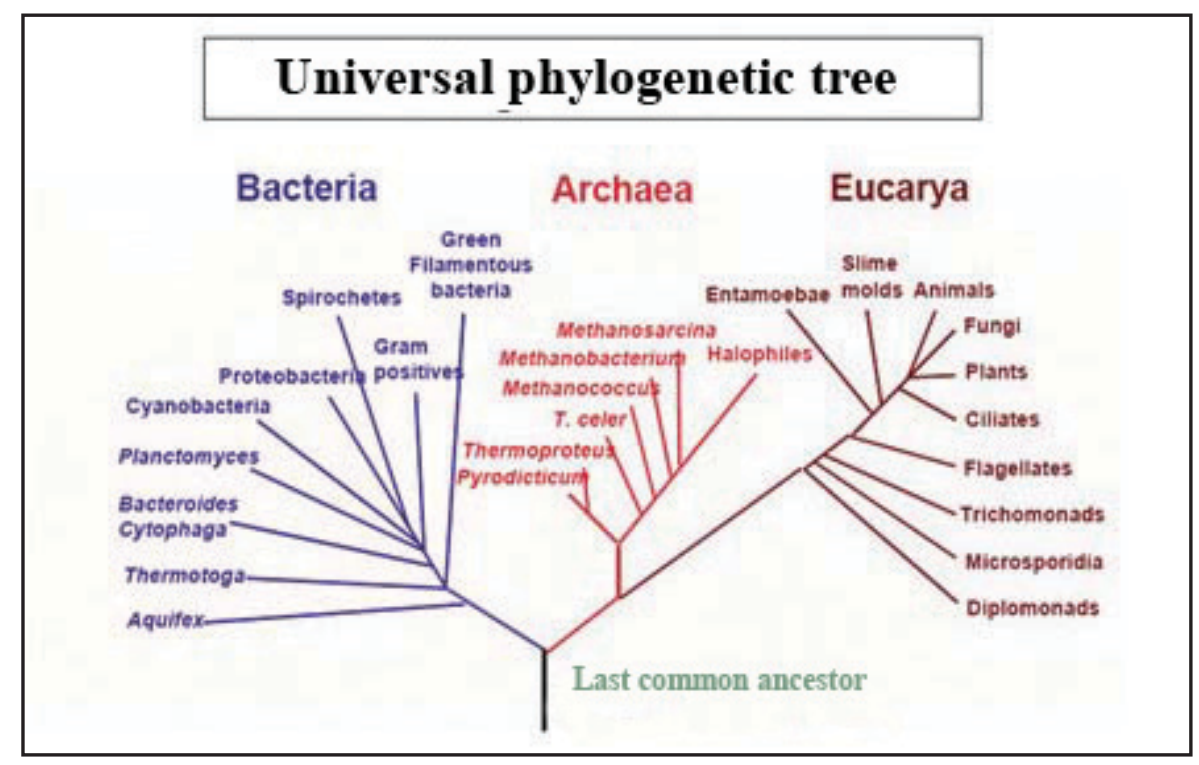

Figure 4 - Universal phylogenetic tree of life based on the 16S Ribosomal RNA, showing the existence of a common ancestor to all life forms. 
Apparently, the formation of the phylogenetic tree was not as simple and linear as presented here. In eukaryotes, the mitochondria (which are responsible for respiration) and the chloroplasts (which are responsible for photosynthesis) seem to have originated from bacteria that invaded the inside of cells, installing themselves in a symbiotic association. ${ }^{4}$ In the same way, primitive organisms might have associated themselves "horizontally" exchanging genetic material. Thus, instead of a single trunk, the phylogenetic tree might have originated from several separate trunks that ended up connecting themselves in three great branches, which later subdivided into secondary branches. Despite that, we will continue to talk about the common ancestor, regardless if it appeared as a single kind of organism or if it was the result of an agglutination of different ancestries.

\section{The progenote (last common ancestor)}

The last common ancestor must have had characteristics shared by all living organisms: reproduction through genes (DNA), protein factories (ribosome and RNA), and mechanisms to repair mistakes in the code, as well as obtaining and storing energy. It must have been more similar to the most primitive organisms (lower branches of the universal tree) than to the most modern ones (tips of the branches). The organisms of the lower branches can stand heat, the thermophiles and hyperthermophiles. They live in temperatures similar to that of boiling water $\left(90-113^{\circ} \mathrm{C}\right)$, such as those found in the deep sea hydrothermal vents and in mud volcanoes such as those in Yellowstone Park (United States). However, there are techniques that allow us to evaluate the formation temperature of nitrogenous bases, and they indicate that the primitive organisms must have originated in environments with moderate instead of extreme temperatures. Another fact that defies the origin of life in high temperatures is its disaggregating effect over RNA, sugars and some aminoacids. At $100^{\circ} \mathrm{C}$ the half-life of several compounds range from seconds to hours. Thus, the hyperthermophiles must have adapted to the high temperatures but they must not have been formed in them. That, along with the complex machinery that they already presented, indicate that they could not have been the first life form.

In the first half of the last century, people imagined that life would have already begun producing its own food (autotrophism ${ }^{5}$ ) like the current organisms that undergo photosynthesis. In photosynthesis, for example, $\mathrm{CO}_{2}$ from the air is absorbed by the cell and, under the action of light and with the use of water, generates a series of organic compounds, mainly sugars such as glucose. In a later stage, they are used to generate energy and to produce structural compounds (body). Animals do not generate, but capture energy produced by other organisms (heterotrophism ${ }^{6}$ ). By means of the oxidation ${ }^{7}$ of sugars, an opposite path from photosynthesis occurs, releasing energy and restoring $\mathrm{CO}_{2}$ to the atmosphere. Near the early 1900's, those processes 
were still being understood and people believed that, without autotrophic organisms, there would be no sources of food in the primitive Earth. However, the appearance of organisms that are already born producing their own food seems implausible today.

In the 1920's, Oparin presented a new idea, which had great developments. He used a scenario of slow and gradual Darwinian evolution, moving from the simpler to the most complex. Starting from the hydrocarbons and from ammonia, other more complex compounds must have been formed, such as carbohydrates and proteins. Similar processes, in a reducing environment, ${ }^{8}$ were proposed by J. B. S. Haldane (1892-1964). After that, the autotrophic scenario lost its impetus, but it still has its advocates today.

\section{The pre-biotic chemistry}

The aminoacids are crucial for life. Nowadays, they are produced by proteins, inside the cells. For life to have appeared, they would have had to be produced by abiotic processes (inorganic). The Oparin-Haldane proposal is that the aminoacids must have been produced from simpler carbonated molecules, in a reducing environment. In the 1950's, Harold Urey (18931981) argued that the Earth's atmosphere, in its origin, was similar to that of the gas giants (Jupiter, Saturn, Uranus and Neptune). They must have supposedly kept their atmospheres almost unchanged due to their great mass (high gravity) and low temperature (distant from the Sun). Rocky planets (Mercury, Venus, Earth and Mars) lost them due to the low gravity and to the proximity to the Sun, which have dissociated the molecules due to the action of the UV rays and that produces a high atmospheric temperature. In the same way that Jupiter and its gaseous partners have atmospheres that are rich in ammonia $\left(\mathrm{NH}_{3}\right)$, methane $\left(\mathrm{CH}_{4}\right)$ and hydrogen $\left(\mathrm{H}_{2}\right)$, the primitive atmosphere of the Earth and other rocky planets must also have been like that.

Urey's hypothesis filled his student Stanley Miller with enthusiasm. He knew Oparin's theory that the aminoacids could be formed by abiotic processes in a reducing atmosphere and decided to test this in laboratory. In 1953, he assembled an experiment simulating the atmospheric processes, in which a gas made up of ammonia, methane and hydrogen went through a chamber where there were electric sparks, after that it was condensed in a water flask and evaporated again, in a continuous cycle. In a few days, a precipitate rich in aminoacids was formed. That result is spectacular and it opened new horizons to understand the origin of life. The experiment is a postcard shown by science teachers as being the demonstration that this is how life originated on Earth, but that is incorrect for two reasons.

The first problem with Miller's experiment is that the Earth's atmosphere was never reducing, at least in the necessary degree to form aminoacids. The countless variants of his experiment, when they take place in neutral environments (between oxidizing and reducing) or lowly 
reducing, never produced significant amounts of aminoacids. Around the 1970's, planetologysts demonstrated that the idea of reducing atmospheres in rocky planets was inconsistent (see Delsemme, 2000). The rocky planets were formed from dry dust, without the covering of water and volatile elements that formed the gas giants. That is why rocky planets have neutral atmospheres (rich in carbon dioxide and nitrogen) and the gas giants have reducing atmospheres (rich in hydrogen, ammonia and methane). The second problem is that the experiments never produced life or anything more complex than aminoacids.

Therefore, how can we explain the fact that the Earth and other rocky planets have water today (although not much compared to bodies further away from the Sun)? The fragments left over from Jupiter's formation and from the other gas giants were spread all over, in the form of comets, and many of them hit the Earth, bringing a great amount of water and carbonated compounds. The formation of the Moon (4.42 B.a.), by the collision with a planetoid the size of Mars with the Earth, cooked the terrestrial crust and vaporized the oceans brought by comets. New incoming comet and meteorite brought more water and carbonated compounds. The heat from the impacts, the greenhouse effect of solar light on the $\mathrm{CO}_{2}$ rich atmosphere and the dissociation of the hydrogenated molecules by UV radiation do not leave much room for a reducing atmosphere (rich in hydrogen).

The discovery of the submarine hydrothermal vents brought new hope of finding that reducing environment. Those places are interesting because they are protected from the impact of meteorites and comets, since they exhale interesting compounds to pre-biotic chemistry $\left(\mathrm{H}_{2} \mathrm{~S}, \mathrm{CO}\right.$ and $\left.\mathrm{CO}_{2}\right)$ and for their thermal energy source (temperatures up to $350^{\circ} \mathrm{C}$ ). If they also exhaled $\mathrm{HCN}, \mathrm{CH}_{4}$ and $\mathrm{NH}_{3}$, they would form a reducing environment and possibly some kinds of aminoacids, although not all necessary to life, due to the disaggregating effect of the high temperature on some of them.

While it is difficult to find a favorable environment to the formation of aminoacids in the primitive Earth, it can be shown that Miller's experiment is widely operational outside of it. Some meteorites (of the chondrite type), such as the one that fell in Murchison, Australia, in 1969, contain a considerable amount of aminoacids (100 ppm - parts per million), and they are of the same types of those produced in Miller's experiment. No wonder that happens, since those celestial bodies were formed in the region of the gas giants, where the protoplanetary disk was rich in hydrogen. Comets are rich in organic compounds $\left(50 \% \mathrm{H}_{2} \mathrm{O}, 1 \% \mathrm{HCN}, 1 \% \mathrm{H}_{2} \mathrm{CO}_{3}\right.$, besides $\mathrm{CO}, \mathrm{CO}_{2}$ and aminoacids) and could have brought a considerable amount of aminoacids in the last stages of Earth's formation. The small fragments, mainly the comet dust, do not generate much heat when they fall, in such a way that the aminoacids might have survived the fall. Today, $\sim 40$ thousand tons/year of 


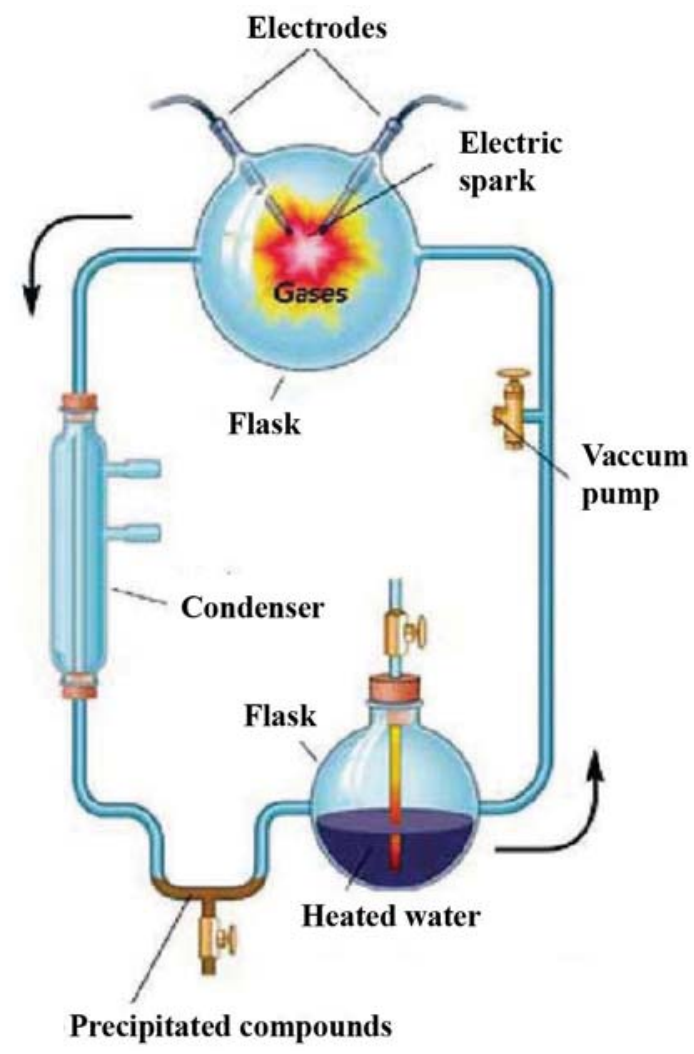

Figure $5-S$. Miller's experiment for abiotic synthesis (inorganic) of aminoacids in a reducing atmosphere, which Urey supposed to have existed in the primitive Earth.

comet dust fall on the Earth and this rate might have been 100-1,000 times higher in Earth's early days. The question that is still hard to answer is if the origin of aminoacids from outside the Earth would have been sufficient to originate life here.

The formation of aminoacids is surprisingly easy in the typical environments of the Universe, rich in molecular hydrogen. So easy that three types of simple aminoacids were detected in interstellar clouds such as the Orion nebula. More complex aminoacids are very hard to be identified, but they might be present due to the huge variety of complex molecules present in interstellar clouds.

Until now, no mechanism or environment that can produce the entire diversity of organic compounds necessary to life could be found. The pre-biotic soup might have had contributions from different processes occurred in the interplanetary environment, in Earth's atmosphere and in the hydrothermal vents. Although the problem of knowing how much each source contributed to the pre-biotic soup is still open, the abiotic origin of organic compounds that are crucial for life, such as the aminoacids, is firmly founded in laboratory experiments and theoretical processes. 


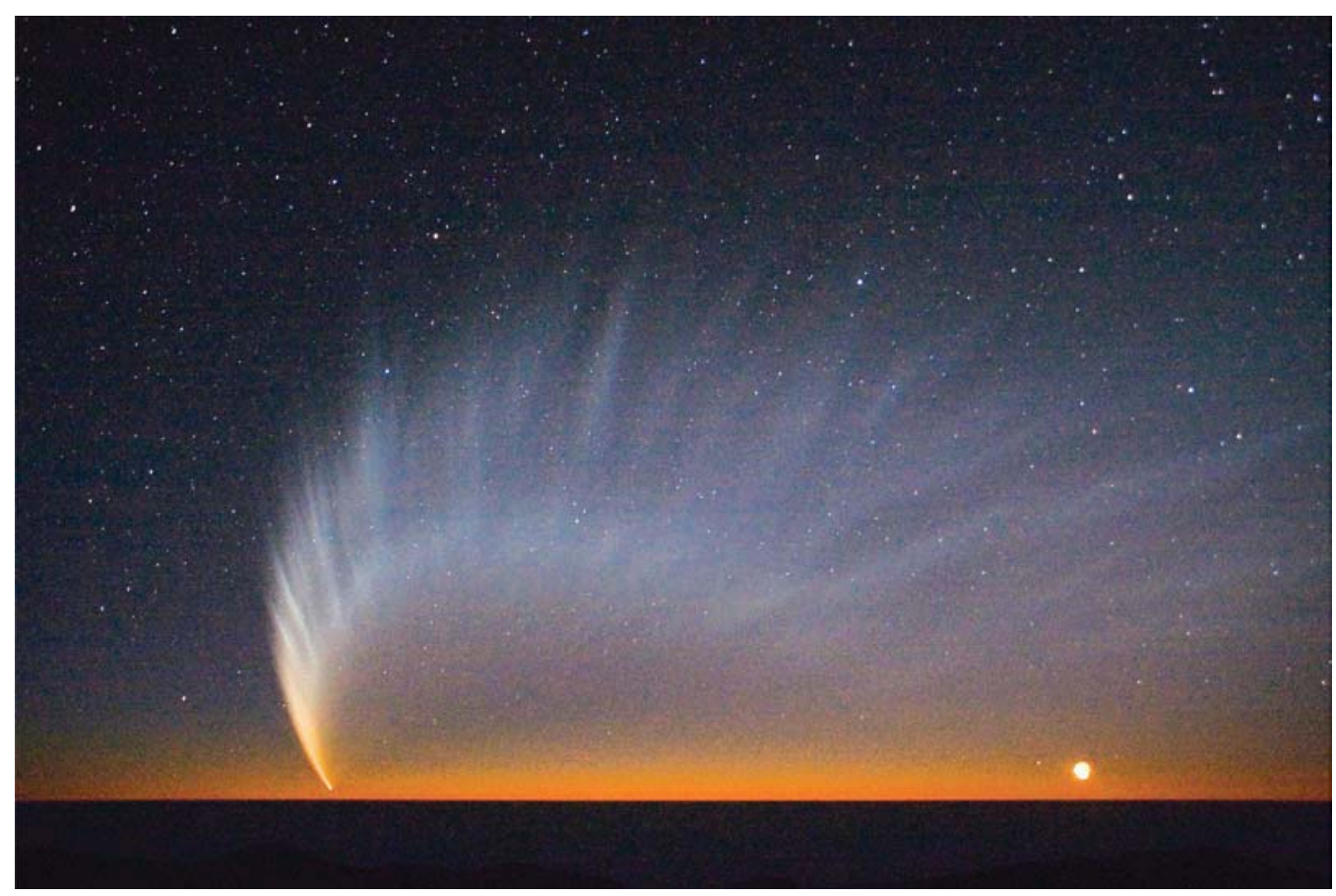

Figure 6 - Comets brought water, biogenic and volatile compounds from the region of the gas giants (Jupiter, Saturn, Uranus and Neptune) to the rocky planets (Mercury, Venus, Earth and Mars) when the solar system reached the age of $\sim 70$ M.a. (ESO).

\section{The origin of the genetic code}

Perhaps the origin of the genetic code is the most challenging step to understand the origin of life. The appearance of a reproduction script corresponds to that of a software, or a natural memory. Which mechanism can do that? The passage from inorganic to life began in a disperse environment and found its focus within the cellular environment. The assembly of smaller molecules in a larger structure must have taken place in an environment characterized by competition and selection. It does not seem that this assembly has occurred simply by means of random processes, otherwise we should have an uninterrupted series of molecules, forming a pyramid with a great quantity of the simple ones forming the base and decreasing in number as the size increases. The DNA molecule has billions of atoms, much more than the other smaller organic molecules. That continuity leap suggests that it has been formed through a specific process, coordinating several elements simultaneously.

The development and reproduction of the current organisms occur by means of a universal genetic code (constituted on nucleic acids) that contains the information about the aminoacid sequences that make up proteins. The nucleic acids are the replication base and the proteins are the metabolism base. 
Roughly speaking, the protein synthesis process is based on the transcription of the information from DNA to messenger RNA and in the translation into proteins. In turn, proteins control both the catalysis and the replication of DNA. That process is so complex that it should have gone through simpler stages in previous phases. How did it begin? Paraphrasing the paradox of the egg and the chicken, the question emerges: what came first, the genetic code or metabolism? There are advocates of both possibilities. There is a hypothesis that RNA was the first active molecule in the origin of life called RNA world. However, RNA is also so complex that there is the hypothesis of a pre-RNA world, but biochemistry as we know gives us no clues about the primeval chemistry and we would have to investigate new scenarios. It is still not clear what kind of molecule might have formed the first genetic material and the stages that might have led to a RNA world are still cloudy.

The second argument admits that metabolism might have appeared before the genetic code. The general idea of that point of view is that it is possible that there is considerable organization in the sequence of chemical reactions themselves, without the existence of a genetic code. However, that view still lacks experimental evidences, since it is unlikely that long polymers and complex reactions may organize themselves in an autonomous manner. Some authors believe that there might be a principle of self-organization that operates in that way.

\section{When did life start?}

People often think that something as complex as life would require certain processes that only occur rarely, demanding extremely long periods of time for them to have any chance to occur. Current data indicate that this is false. Let's take a look at the first few geological eras of the Earth. Isua (in Greenland), one of the oldest rocky formations, is about 3.8 B.a. Although it does not contain fossil organisms, it has some indications of contamination by biological activity. The graphite found in it contains ${ }^{13} \mathrm{C}$ (a variety of the carbon atom with six protons and seven neutrons) compared to the lighter isotope ${ }^{12} \mathrm{C}$ with figures that are typical of organic material, such as the one found in current vegetable remains. Until now, no other explanation has been found, except for photosynthesis to explain that carbon anomaly.

Another datum that points to photosynthesis in remote times is the huge deposits of iron oxide (called banded iron formation - BIF), the oldest of which are $\sim 3.7$ B.a. At that time, there was no free oxygen in the atmosphere, as indicated by the existence of pyrite and uraninite. Oxygen might have been released in the oceans by the activity of photosynthetic algae and consumed locally, oxidizing the iron. If the Isua rocks and the oldest BIFs indicate the existence of life, it must have appeared before 3.8 B.a. ago since the complex process of photosynthesis must not have been the first form of energy production. The common ancestor must have appeared before that. 
Still another reason to move back the appearance of the progenote to before 3.8 B.a. is that the following three hundred million years seem to be a short time for life to have reached the complexity level of the cyanobacteria, related to the organisms that formed the stromatolites. But we cannot move back the origin of life much before that time. The Earth was formed 4.6 B.a., and at 4.46 B.a. it already had a solid crust, water had poured from the clouds to form oceans and the atmosphere had an acceptable temperature. But in the first 700 million years it was hit by a dense meteor shower. Some of the fragments were hundreds of kilometers in size. Such a collision would vaporize the oceans and would warm the atmosphere so much that it would take more than one thousand years to rain again. If life already existed on Earth at that time, it would have been destroyed, not once, but many times. It could only have settled in a stable way after the end of the sterilizing meteor showers, that is, less than 3.9 B.a. This leaves a window of $<100$ million years for life to begin from zero and reach the stage of energy production by photosynthesis. If we prefer to reject the differential of ${ }^{13} \mathrm{C}$ or the BIFs as an indication of life, the time range for life to have been formed and evolved to the complexity level of the cyanobacteria raises to mere 400 M.a. In the following 3.5 B.a. life increased its diversity, but there were no complexity leaps as large as the first one, from the inorganic to the living. For that reason, the time window of hundreds of millions of years is small and indicates that this leap is not so hard or unlikely for nature.

The window for the origin of life, if it in fact began on Earth, might be much shorter than the four hundred million years indicated above. A very short time scale could be obtained from the fact that chemical reactions that produce large molecules (polymers) are reversible. In a matter of days or months, most of them would revert to simpler components in an aquatic environment. That could be avoided if the large molecules were removed from the liquid environment as soon as they were formed. That scenario would work if life had appeared in the continental shelves instead of deep in the oceans. In the beginning, the lithospheric plates ${ }^{9}$ were still under water, and the most solid indications are that life has appeared deep in the oceans. Another way to avoid reversibility would be to enclose the macromolecules in membranes, such as the cellular walls. At that time there were no cells such as the current ones, but there might have been membranes formed by inorganic processes. Oparin suggested that coacervates,${ }^{10}$ formed spontaneously by polymers in water, must have constituted the membrane of a protocell. Although they are actually formed in laboratory, they are very unstable. There are other possible kinds of membrane formation. For example, the proteinoids ${ }^{11}$ that Sidney Fox synthesized in laboratory.

Even after escaping from the reversibility, the components of primitive life found other fatal pitfalls. One of them is the hydrothermal vents that exist deep in the oceans. They recycle a volume equal to that of 
the current oceans in ten million years. However, when the interior of the Earth was hotter, that process was much stronger and the recycling times were much shorter. Water gets out of the hydrothermal vents at $\mathrm{T}>350^{\circ} \mathrm{C}$, being completely sterilized.

The shorter the time scale, the simpler must have been the process of the origin of life. On Earth, it was installed so early and so quickly that it seems to be a mere by-product of the planetary formation. That opens huge perspectives that it might also have appeared in other planets, which in our galaxy alone are expected to be over the range of trillions. Within the visible volume of the Universe there are around one hundred billion galaxies like ours, increasing the number of planets to more than $10^{23}$. The fact that the origin of life is such a hard issue to understand should not lead us to the mistake of assuming that it is also hard for nature to accomplish it. The window for the formation of life on Earth is so narrow that some people prefer to believe that it has arrived here already formed (panspermia hypothesis). The comfort obtained by increasing the time window to ten billion years and multiplying the variety of physical and chemical situations by a countless number of planets gets lost due to the huge cosmic isolation of the celestial bodies and to the lack of feasible transport mechanisms of living organisms from one to the other. Transportation is only feasible for

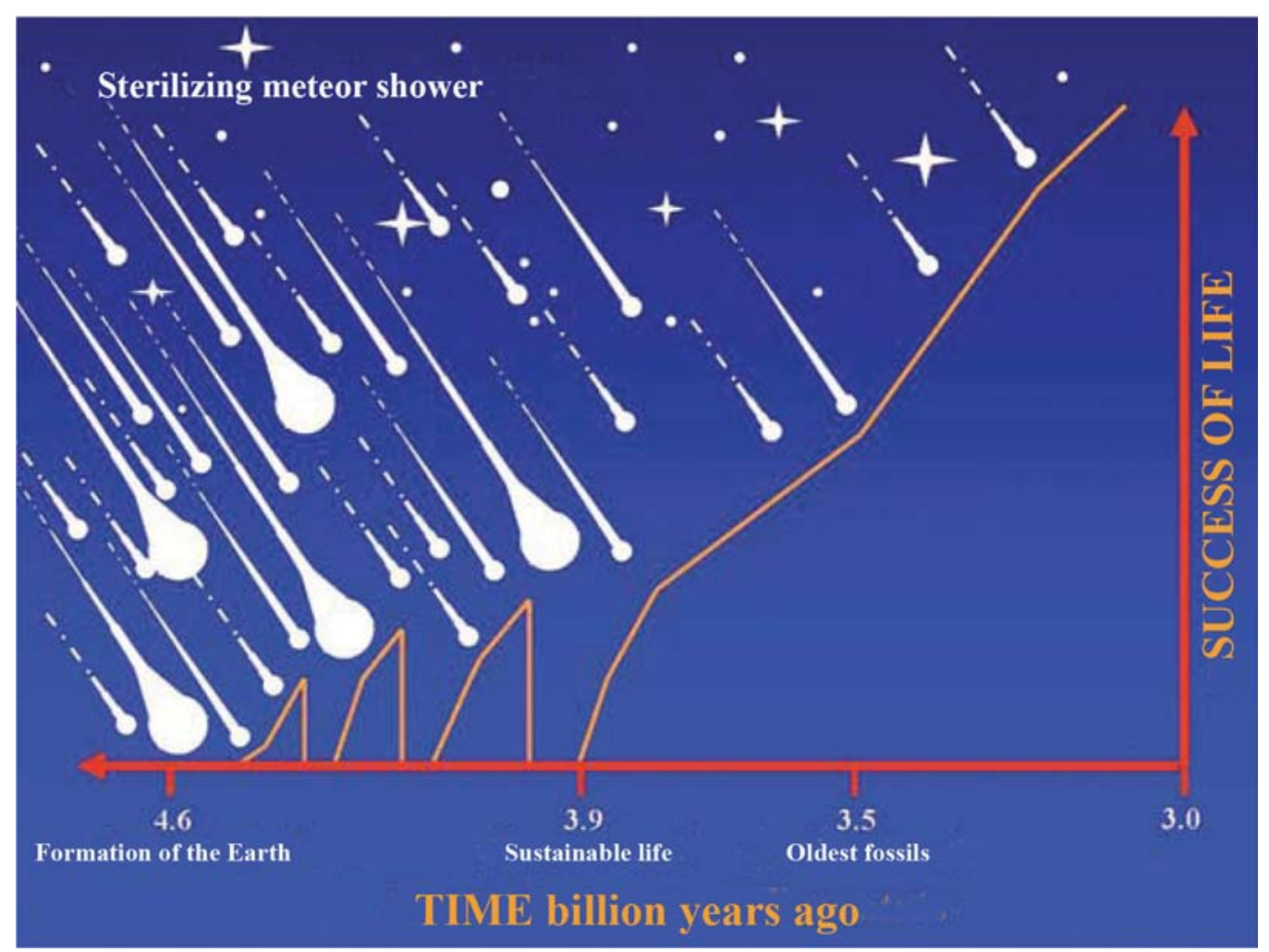

Figure 7 - Establishment of life on Earth after $\sim 700$ million years old, when the sterilizing meteorite shower came to an end. 
planets that are close to one another, such as between the Earth and Mars. The problem of the origin mechanism is transferred from here to another planet, but its solution does not become any easier.

\section{Perspectives of life outside the Earth}

The study of life in the astronomical context is significant for several reasons. The most crucial of them is that we can never have a general theory of life while we know only the terrestrial specimen. Let's take physics as a paradigm. It has general theories because there are countless situations in which they can be applied. Also, one can verify how each parameter varies from one situation to another. The multiplicity of situations enable us to make predictions and to test the theory empirically. Only the discovery of other life specimens, formed outside the Earth, will allow us to see what is fundamental and what is secondary in the phenomenon. Another reason is that the information on the physical and chemical conditions of the Earth, at the time when life was established here, were lost forever. The observation of other celestial bodies allows us to review the past, since everything we see in space are events that took place in the past, the more distant in space, the more remote in time. Light goes through space and, in the same way as the rock that goes through time, brings fossil records. In space there are countless planets in several stages of formation that allow us to review the evolutionary stages by which the Earth has gone through, as if it was a journey through time. Another reason to look at the sky is simply to test the basic assumption of evolutionism that assumes that life is a natural fact, emerging as part of the processes of energy dissipation and transformation of matter. Any other planet in physical conditions equal to those of the Earth would have had the same probability to generate life.

Until now, the only enterprise to look for life outside the Earth was by means of capturing radio signs that could have been sent by extraterrestrial civilizations. Although we are sympathetic to any methodic effort, those projects were eventually excluded from the agenda of the governmental funds for understandable reasons. First, there is no testable theory about the probability of evolution having produced communicating beings such as ourselves in other places, or about their decision to apply resources to tell the entire Universe that they exist, or about the inevitability that communication is made by means of radio waves, or still about how long a technological civilization that requires so many resources can survive without self-destruction. Second, is the modus operandi of the current scientific production. The number of variables involved in radio listening is so great that it is impossible to offer to the funding agencies an expected time frame for results to be obtained. To which star should we point, for how long should we keep listening, in what frequencies should we monitor the signals, what kind of signs derive from language and which 
are natural emissions? How many students would be convinced to devote their lives to a project that could take millennia to present a result, receiving scholarships for only a few years?

The current projects to search for life are based on a classic scientific procedure. They are well fundamented and have captured the imagination of the tax-payer in such a way that the resources already permit a solid advance. This will certainly be one of the great themes of the $21^{\text {st }}$ century. The results are expected within a time frame of one or two decades and we might be alive to witness them. What will we look for? Simply the most common life form that we know on Earth that has been living in our planet for the longest time: microbes. Would it not be a lack of imagination to restrict the search to the trivial that we know? There are excellent reasons for the restriction. First, it is no use to look for something that cannot be identified. Second, microorganisms contaminate the planets' atmospheres with molecules that are easily identifiable at great distances, such as ozone $\left(\mathrm{O}_{3}\right)$, for example. An atmosphere with ozone layer is unthinkable without a large scale photosynthetic activity. Due to its high chemical reactivity, oxygen is firmly bonded in molecules and, to be released, it needs an agent that goes in the opposite direction of the chemical potential. That indicates that there is a process that acts against the thermodynamic equilibrium (locally), which is one of the characteristics of life. Besides, ozone is decomposed by the ultraviolet light of the Sun, and the maintenance of a layer in the atmosphere implies a constant replacement of free oxygen, which would indicate a continuity of the source, in other words, reproduction - another characteristic of life. In the rocky planets, methane operates exactly in the same way as ozone, but indicating heterotrophic activity instead of autotrophic. On Earth, the methane layers are produced by anaerobic bacteria in embankments, inside animals' intestines or in decaying organic matter in swamps. In the case of gas giants planets, methane has an abiotic origin and its presence does not indicate biological activity.

Third, it is very likely that "life as we know it" exists. Not only did it appear on Earth right in the beginning, but it kept expanding even when it suffered the action of global catastrophes such as volcanism, freezing and the impact of great meteors. Life is closer to an invading and resistant plague than to something that is improbable and delicate. The chemical elements that it requires are among the most abundant in the Universe; and, when it reached two billion years old ( 12 B.a.), the periodic table already had elements in all its squares. At that time, there was already a huge amount of water and complex molecules could start to be formed, as one can observe in the interstellar clouds. The formation of the planets followed that of the stars, since they are part of the same process. The peak of stellar formation was reached $\sim 10$ B.a. ago, in such a way that typical 
planets are old. That does not mean that life is swarming all around the Galaxy, but that "life as we know it" is quite likely to exist and to be found by means of contaminations in the planetary atmospheres.

The search for microscopic life in the solar system took place in a very limited manner in Mars. The interest in Mars is because it is relatively close and because it presented favorable conditions to life in its beginning. It had shallow oceans throughout hundreds of millions of years, meteor impacts were less brutal than on Earth due to its lower gravity and the atmospheric temperatures seem to have been milder than here. Everything put together, Mars presents a greater favorable factor for the appearance of life than the Earth and with a longer time window. The fact that the planet froze more than 3.5 billion years ago indicates that, if life did exist there, it was interrupted in the very outset, unless it has been transplanted here by the countless meteorites ( 40 billion tons) that arrived here. If we find life there of the same kind as Earth's, that will not become a second point to support the generalization of the theory of life. We will not be able to exclude the possibility that we are Martians or that they are Terrestrials, since the probability of contamination in both ways is not negligible.

Jupiter's Moon, Europa, is attractive because it has a liquid water sea with hydrothermal vents (beneath an icy surface), in a situation not very different from the one people consider today as characteristic of the beginning of life on Earth. Its advantage as compared to Mars is the extremely low probability of contamination from the Earth. But making a hole that is tens of kilometers deep in Europa's icy surface and searching for microbes in a greater volume of water than that of the Earth's ocean is a currently economically unfeasible enterprise. There are no other places in the solar system that offer such good perspectives as Mars and Europa for life to have appeared.

Anyway, the solar system is a bit irrelevant for a general theory of life, since we need several different examples. In order to search many planets, we must look at the stars, around which there must be trillions of them, in our galaxy alone. The current instruments can only detect planets indirectly, by means of the gravitational reaction of the host star, and that is limited to atypical cases (very large planets and very close to the central star). Even so, more than 200 extra-solar planets are already known. All of them are uninteresting for life, since they are gas giants that recycle their atmospheres between the temperatures of thousands of degrees in the interior to $-180^{\circ} \mathrm{C}$, on top of the clouds.

The Holy Grail of the search for life is hidden in the rocky planets such as our own. Is it another lack of imagination? Perhaps. On the one side, we have still not freed ourselves from the image of Mother Earth, welcoming for life. But, on the other hand, there is the fact that the 


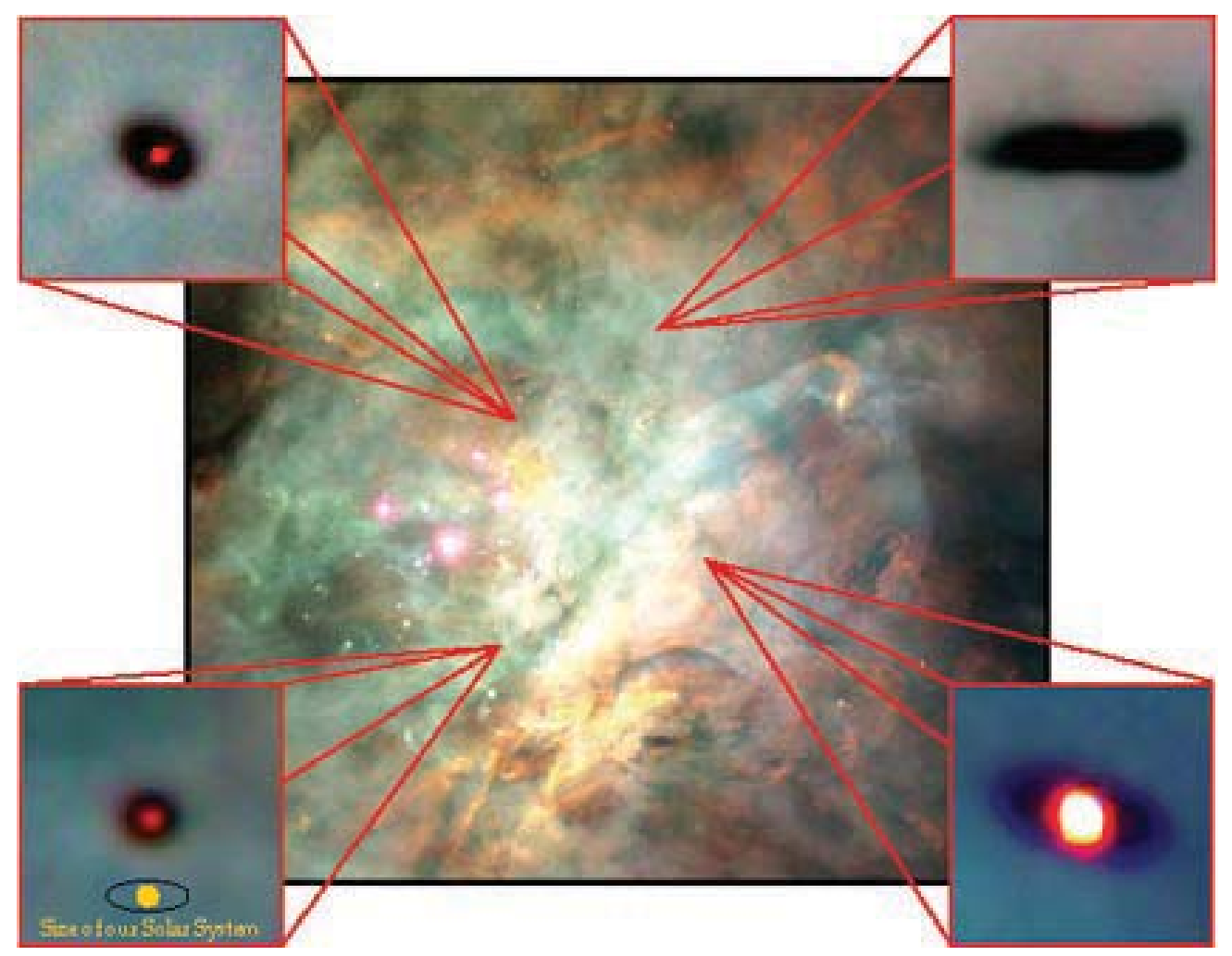

Figure 8 - Planetary systems being formed in the Orion Nebula, showing disks of protoplanetary dust (dark stains). They allow us to review how to the formation of the solar system took place. (Nasa).

contamination by products of the biological activity is easily visible in the tenuous atmospheres of the rocky planets, since they are very rarefied. The current telescopes are way beneath the necessary sharpness to take pictures of rocky planets around other stars. But that is a simple technical issue that can be solved with human and financial investments. The financial resources are appearing. It will be necessary to increase the resolution of the telescopes by a factor of one thousand (the leap was of a 10 factor in the last four-hundred years since Galileo). After that, we must screen the light photons that come from the planet since for every billion photons of the host star only a few from the planet enter the telescope. It will be necessary to turn off the star, without interfering in the light of the small planet, located fractions of arcseconds away from it. The instruments for that are already being developed, and the first direct detections are expected to happen in less than two decades. Then we will only have to pass the light of the rocky planets through the spectrograph and look for the signature of ozone and methane. 


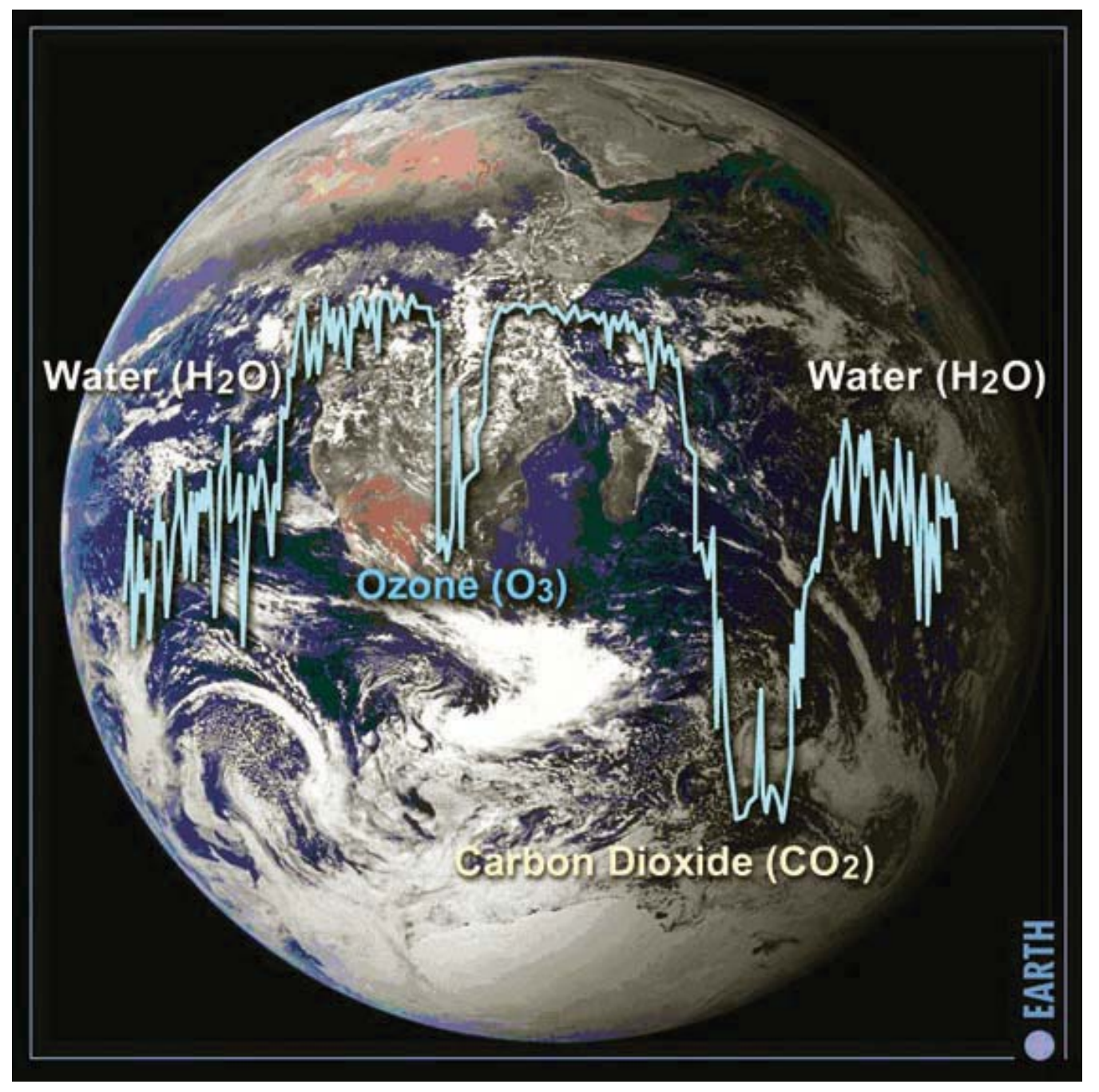

Figure 9 - Earth spectrum in the medium infrared, collected from space, showing a molecular band of ozone in the atmosphere. That is considered an undeniable sign of biological activity (photosynthesis). (Project Darwin- ESA)

Meanwhile, new generations of researchers related to those projects will be able to find out other more characteristic signs of biological contamination different from those presented here. After screening an extensive list of planets, we may have sufficient statistics to know if the Universe is as biophilic as it seems today. We might not find any sign of life (for the great delight of the creationists who oppose the evolution theory), but the only choice of the scientific progress is to produce tests that may go against the theoretic expectations. If there are positive detections, then it will be the beginning of the study of each specific kind of life and it would be fictional to speculate which will be the techniques used for that 
to happen. Therefore, a general theory of life is not expected so soon, but if anything is clear it is that the technological and scientific advances have always overcome the expectations and arrive before expected.

\section{Notes}

1 Stromatolite: Rocky formation made up of calcium carbonate, deposited from dead algae in the shallow waters of the oceans.

2 Monomers: Molecular subunits that, once assembled, form long chains, called polymers.

3 Genomic Analysis: Mapping of the sequence of nitrogenous bases pairs (AG -adenine and guanine; CT - cytosine and thymine) that make up the DNA of a given organism.

4 Symbiotic Association: When two organisms assemble with mutual advantages. For example, mitochondria generate energy for the cell and, in turn, the cell offers protection and food to it.

5 Autotrophism: When an organism produces its own food, such as in the case of plants.

6 Heterotrophism: When an organism nourishes itself with the energy generated by others, such as in our case.

7 Oxidizing Substance: The one that receives electrons, such as those rich in oxygen. Examples of oxidation: rustiness, respiration.

8 Reducing Substance: The one that donates electrons, such as those rich in hydrogen.

9 Lithospheric Plates: The superficial layers of the Earth that constitute the continents. They are made up of plates that collide with one another, producing earthquakes.

10 Coacervates: Colloidal systems (closed structures) generated in polymer solutions (molecules with long carbon chains).

11 Proteinoids: Large molecules, similar to proteins, made up of aminoacids, but of abiotic origin.

12 arcsecond: Angle size measurement, a subdivision of the degree. The human eye can only see objects that are larger than $\sim 120$ arcseconds.

\section{Bibliography}

DELSEMME, A. Cometry Origin of the Biosphere. Icarus, v.146, p.313-25, 2000.

JAKOSKY, B. The search for life on other planets. Cambridge: Cambridge University

Press, 2000.

LAZCANO, A. The Origins of Life. Natural History, p.36-41, Feb. 2006. 
McCLENDON, J. H. The Origin of Life. Earth Science Reviews, v.47, p.71-93, 1999. MENCK, C. F. M.; DE OLIVEIRA, M. C. Biologia molecular e evolução. Editor S. R. Matioli. Ribeirão Preto: Holos, 2001.

SCHOPF, J. W. Craddle of life. s. 1.: Princeton University Press, 1997. (Ed.) Life's origin. s. 1.: University of California Press, 2002.

\begin{abstract}
We present a historical perspective about the ideas concerning the origin of life. After displaying the main topics necessary for understanding life's origin, the main characteristics of the present life forms and their relationships are shown, suggesting a common ancestor. The conditions for prebiotic chemistry in terrestrial or interplanetary environments are reviewed. We put in context the arguments about the early origin of replicators versus metabolism. The very narrow window for life settlement in the early Earth is stressed, pointing to the likelihood of life arising in other places in the Universe. Finally, we present the cornerstones of current search for life outside our planet.
\end{abstract}

KEYWORDS: Life, Origin of life, Evolution.

Augusto Damineli is full professor of the Astronomy Department of the Astronomic and Geophysical Institute of the University of São Paulo (IAG-USP).

@ - damineli@astro.iag.usp.br.

Daniel Santa Cruz Damineli has a bachelor degree in Biology from the University of São Paulo (USP) and is a postgraduate student at São Paulo State University (Unesp) - Rio Claro. @-danieldamineli@gmail.com.

This text has been translated by Rodrigo Sardenberg. The original in Portuguese - "Origens da vida" - is available at http://www.scielo.br/scielo.php?script=sci_ issuetoc\&pid=0103-401420070001\&lng=pt\&nrm $=$ iso.

Received on 10.5.2006 and accepted on 11.6.2006. 